\title{
Laparoscopic Undo of Fundoplication with Roux-en-Y Gastric Bypass in a Morbidly Obese Patient with Prior Nissen's Fundoplication: A Video Report
}

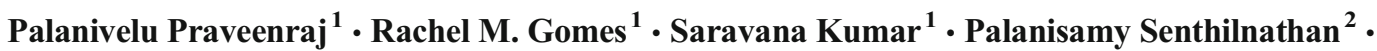 \\ Ramakrishnan Parthasarathi $^{2} \cdot$ Subbiah Rajapandian ${ }^{2} \cdot$ Chinnusamy Palanivelu $^{2}$
}

Published online: 18 November 2015

(C) Springer Science+Business Media New York 2015

\begin{abstract}
Background Roux-en-Y gastric bypass (RYGB) may be a better option for morbidly obese patients with gastroesophageal reflux (GERD) for long-term reflux control. It is recommended after fundoplication if a patient is morbidly obese with GERD with failed fundoplication or if bariatric surgery is planned with a prior successful fundoplication (Kim et al., Am Surg 80(7):696-703, 2014; Kambiz Zainabadi, Surg Endosc. 22(12):2737-40, 2008). Complete takedown of the wrap to avoid stapling over the fundoplication creating an obstructed, septated pouch is needed (Kambiz Zainabadi, Surg Endosc. 22(12):2737-40, 2008). The aim of this video was to demonstrate the technical aspect of dissection and undo of Nissen's fundoplication followed by performance of a RYGB in a morbidly obese patient with GERD with prior successful Nissen's fundoplication opting for bariatric surgery after a year.

Methods We present a case of a 50-year-old woman with a BMI of 36.14 with previous laparoscopic Nissen's fundoplication for severe GERD (controlled after surgery) and a prior laparoscopic intraperitoneal onlay mesh repair who presented for bariatric
\end{abstract}

Electronic supplementary material The online version of this article (doi:10.1007/s11695-015-1957-3) contains supplementary material, which is available to authorized users.

Palanivelu Praveenraj

praveenraj@me.com

1 Department of Bariatric Surgery, GEM Hospital and Research Centre, 45, Pankaja Mill Road, Gem Hospital and Research Centre, Coimbatore 641045, India

2 Department of Surgical Gastroenterology, GEM Hospital and Research Centre, 45, Pankaja Mill Road, Gem Hospital and Research Centre, Coimbatore 641045, India surgery 1 year after fundoplication. She was successfully treated by laparoscopic undo of the fundoplication with RYGB.

Results In this multimedia high-definition video, we present stepby-step the laparoscopic undo of a Nissen's fundoplication followed by RYGB. The procedure included lysis of all adhesions between the liver and the stomach, dissection of the diaphragmatic crura, complete takedown of the wrap, repair of the hiatal hernia, creation of a gastric pouch, creation of an antecolic Roux limb, gastrojejunal anastomosis, and jejuno-jejunal anastomosis.

Conclusion Laparoscopic RYGB after fundoplication in morbidly obese patients with GERD is a technically difficult but feasible option.

Keywords Morbid obesity · Gastroesophageal reflux disease $\cdot$ Fundoplication $\cdot$ Undo fundoplication $\cdot$ Bariatric surgery $\cdot$ Gastric bypass

Compliance with Ethical Standards Informed consent was obtained from all individual participants included in the study. The study has been approved by the appropriate institutional committee.

Conflict of Interest The authors declare that they have no competing interests.

Financial Support None

\section{References}

1. Kim M, Navarro F, Eruchalu CN, et al. Minimally invasive Roux-en$\mathrm{Y}$ gastric bypass for fundoplication failure offers excellent gastroesophageal reflux control. Am Surg. 2014;80(7):696-703.

2. Kambiz Zainabadi APC. Laparoscopic revision of Nissen fundoplication to Roux-en-Y gastric bypass in morbidly obese patients. Surg Endosc. 2008;22(12):2737-40. 